\title{
Histological Study on the Protective Effect of Green Tea Extract on the Liver of Rats Exposed to Ketamine
}

\section{Lamiaa Ibrahim Abd El-Fattah and Dalia Ibrahim Ismail*}

Department of Histology, Faculty of Medicine, Cairo University, Egypt

\begin{abstract}
Background: Green tea intake is accompanied with a lower incidence of cancer, cardiovascular disease, and neurodegenerative disorders; hence green tea extract is included in multivitamins and other dietary supplements.

Aim of the work: Studying the effect of ketamine administration on the liver of adult male albino rats and demonstrating the possible protective role of green tea extract.

Material and methods: Forty adult male albino rats were divided into four groups, ten rats each. Group I: control group. Group II: rats received green tea extract orally $(50 \mathrm{mg} / \mathrm{Kg} /$ day $)$ for five weeks. Group III: rats were injected intraperitoneally with ketamine $(100 \mathrm{mg} / \mathrm{kg} /$ day $)$ for two weeks. Group IV: rats received green tea extracts orally ( $50 \mathrm{mg} / \mathrm{Kg} / \mathrm{day}$ ) for three weeks, and then continued for further two weeks concomitantly with intraperitoneally injected ketamine (100 mg/kg/day). Serum levels of AST, ALT, GSH and MDA were measured. Liver sections were subjected to $\mathrm{H} \& \mathrm{E}$, iNOS immunohistochemical stain, and toluidine blue stain and transmission electron microscopic examination. Followed by statistical analysis of biochemical and morphometric results.

Results: In group III, serum liver enzymes and oxidative stress indicators together with positive area of iNOS immunohistochemical stain were significantly increased as compared to control. In liver sections of ketamine treated group, hepatocytes showed signs of degeneration and multiple cytoplasmic vacuoles of variable sizes with dilated blood sinusoids. At ultrastructural level, nuclei with irregular outline, dilated and discontinued RER with empty spaces within the cytoplasm of hepatocytes were recorded. Interestingly, the picture was comparable to control with concomitant use of green tea extract.
\end{abstract}

Conclusion: Green tea extract has a hepato-protective effect in rats exposed to ketamine.

Keywords: Liver; Ketamine; Green tea; iNOS; Electron microscopy

\section{Introduction}

Ketamine is an anaesthetic and analgesic frequently indicated for surgical anesthesia in pediatric patients, treatment of chronic pain and brief anesthesia requirements like wound dressing changes. It is also increasingly abused for recreational purposes. Ketamine is extensively metabolized in the liver by microsomal enzymes and excreted prominently in urine. Its metabolites together with the ketamine itself have been reported to damage hepatocytes and other cells of liver [1].

Many studies have shown that the polyphenolic fractions isolated from green tea inhibit oxidant stress and possess anti-inflammatory activity [2]. They have been reported to prevent liver toxicity induced by a number of hepatotoxicants in animal models including: 2-nitropropane, carbon tetrachloride and acetaminophen. Also, dietary green tea has been shown to prevent fatty liver disease in both dietinduced and genetic animal model [3].

Consequently, the present work aimed at studying the toxic effect of ketamine on the liver, together with the possible hepatoprotective role of green tea extract; this was done using biochemical, histological and immunohistochemical methods.

\section{Materials and Methods}

\section{Materials}

Drugs: Ketamine: Tekam, supplied as $10 \mathrm{mg} / \mathrm{ml}$ of $10 \mathrm{ml}$ vial, from Alhekma co. Egypt.

Green tea extract: Multi -Treat, supplied as tablets of $300 \mathrm{mg}$, in a box of 20 tablets, from MEPACO - Egypt. Tablets were crushed; the required dose weighed using a digital scale and dissolved in distilled water.

Animals: The current study was accomplished on forty adult male albino rats weighing 150-170 gm (mean \pm SD: $162 \pm 3.65$ ). They were obtained from the animal house of Faculty of Medicine, Cairo University. The rats were housed in temperature controlled rooms $\left(25^{\circ} \mathrm{C}\right)$ with constant humidity $(40-70 \%)$ and $12 \mathrm{~h}$ light/dark cycle prior to use in experimental protocols. Rats were fed a standard pellet diet and allowed free access to water throughout the study.

Experimental design: All procedures were carried out in strict compliance with the guidelines of Kasr Al-Ainy medical school, Animals Ethics Committee on the care and use of laboratory animals. Rats were divided into four equal groups as follows:

- Group I (Control group): Ten rats received distilled water orally for three weeks and continued with intraperitoneally injected saline for another two weeks.

*Corresponding author: Dalia Ibrahim Ismail, Department of Histology, Faculty of Medicine, Cairo University, Egypt, Tel: 01005050870; E-mail: drdaliaibrahim@hotmail.com

Received June 25, 2015; Accepted July 15, 2015; Published July 17, 2015.

Citation: Abd El-Fattah LI, Ibrahim Ismail D (2015) Histological Study on the Protective Effect of Green Tea Extract on the Liver of Rats Exposed to Ketamine. J Cytol Histol 6: 349. doi:10.4172/2157-7099.1000349

Copyright: (C) $2015 \mathrm{Abd}$ El-Fattah LI, et al. This is an open-access article distributed under the terms of the Creative Commons Attribution License, which permits unrestricted use, distribution, and reproduction in any medium, provided the original author and source are credited. 
- Group II (Green tea group): Ten rats received green tea extract (50 $\mathrm{mg} / \mathrm{Kg} /$ day) [4], orally via gastric tube once daily for five weeks.

- Group III (Ketamine group): ten rats were intraperitoneally injected with ketamine $(100 \mathrm{mg} / \mathrm{kg} /$ day $)$ for two weeks divided in two daily doses [1].

- Group IV (Green tea+Ketamine group): ten rats received green tea extract $(50 \mathrm{mg} / \mathrm{Kg})$ orally via gastric tube once daily for three weeks before ketamine administration and continued for two weeks concomitant with ketamine $(100 \mathrm{mg} / \mathrm{kg})$.

\section{Methods}

Biochemical study: At the end of the experiment, just before scarification, blood samples were taken from retro-orbital sinus in collecting heparinized capillary tubes to measure the liver enzymes level (ALT: alanine transaminase and AST: aspartate transaminase), and the oxidative stress indicators (GSH: Glutathione and MDA: Malonyldialdehyde) at the Biochemistry Department, Faculty of Medicine, Cairo University.

Histological study: Animals from all groups were sacrificed following ether anaesthesia.

Light microscopic study: Specimens of liver were fixed in $10 \%$ formol saline for light microscopic examination. Paraffin-embedded sections were cut at 5-7 $\mu \mathrm{m}$ thickness and subjected to:

- Hematoxylin and eosin stain [5].

- Immunohistochemical staining using the avidine-biotin peroxidase complex (ABC) technique for detection of iNOS antigen [6]: using anti iNOS antibody which is a rabbit polyclonal antibody (Thermo scientific, Walthman, Massachausetts, USA). The ABC technique was carried out as follows: The primary antibody was applied for 60 minutes, and then sections were incubated with the secondary antibody (a biotinylated antiserum to rabbit/mouse immunoglobulin). The reaction was visualized by a chromogen (3,3-diaminobenzidine tetrahydrochloride). Tissue sections were counterstained with Mayer's hematoxylin. iNOS positive cells show brown cytoplasmic deposits. Positive tissue control was sections of lung immunostained for iNOS. Negative control was liver sections processed with adding phosphate buffered saline (PBS) instead of applying the primary antibody.

Electron microscopic study: Small pieces of liver tissue $1 \mathrm{~mm}^{3} \mathrm{each}$, from all groups were prepared for semithin and ultrathin sectioning. Specimens were immersed in $2.5 \%$ phosphate-buffered gluteraldehyde ( $\mathrm{pH}$ 7.3) for $24 \mathrm{~h}$, postfixed in $1 \% \mathrm{OSO}_{4}$ for $2 \mathrm{~h}$, and dehydrated in graded concentrations of alcohols. After immersion in propylene oxide, the specimens were embedded in epon resin. Semithin $1 \mu \mathrm{m}$ sections were stained with $0.5 \%$ toluidine blue inborax and examined under a light microscope. Ultrathin sections were cut at $50 \mathrm{~nm}$ thickness and mounted on nickel grids and stained with uranyl acetate and lead citrate [7]. Specimens were examined and photographed using transmission electron microscopy SEO (Sunny Electron Optics) model PEM-100 (Russian), at 75 KV ( TEM of Military Medical Academy, Cairo, Egypt).

Morphometric study: Using Leica Qwin 500 LTD image analysis (Leica image system Ltd.; Cambridge, England): mean area percentage of iNOS positive immunoreactivity was measured at $400 \mathrm{X}$ magnification using the color detect menu, in relation to a standard measuring frame.
It was measured in 10 non overlapping fields for five slides for each animal.

\section{Statistical analysis}

This was performed for serum liver biochemical parameters and for the morphometric results, using SPSS (Statistical Package for Social Sciences) version 16 (SPSS Inc., Chicago, IL, USA). The statistical analysis included standard error mean SEM and analysis of variance ANOVA test, followed by post-hoc Tukey test. The differences were considered significant when $P$ value was less than 0.05 [8].

\section{Results}

\section{Biochemical results}

Statistical analysis of the estimated serum values revealed a significant increase $(\mathrm{P}<0.05)$ in (AST, ALT, and MDA), and a significant decrease in GSH in ketamine treated rats (group III) as compared to other groups. Values of groups II and IV were comparable to control (Table1).

\section{Histological results}

\section{Light microscopic examination:}

Haematoxylin and eosin stained sections: Examination of liver sections from group I (control group) showed central vein in the middle of the classic hepatic lobule, with radiating cords of hepatocytes enclosing blood sinusoids in between. The hepatocytes exhibited acidophilic cytoplasm and central rounded vesicular nuclei; most of the cells were mononucleated, while some were binucleated. Von Kupffer cells exhibiting dark nuclei were demonstrated (Figures 1A and 1B). Sections from group II (green tea group) revealed histological appearance comparable to that of the control group (Figures 1C and 1D). Sections of rat liver from group III (Ketamine group) demonstrated congested central vein. Hepatocytes showed cytoplasmic vacuolization and some of them showed small dark nuclei. Deeply acidophilic fragmented cytoplasmic bodies were also noted (Figures $1 \mathrm{E}$ and $1 \mathrm{~F}$ ). Liver sections from group IV (Ketamine and green tea group) appeared comparable to that of the control group. However, some hepatocytes showed minimal cytoplasmic areas of vacuolization. Others exhibited deeply acidophilic cytoplasm and small nuclei (Figures $1 \mathrm{G}$ and $1 \mathrm{H}$ ).

iNOS immunohistochemically stained sections: Sections of rat liver from both groups I and II showed positive iNOS immunoreactivity within the cytoplasm of some hepatocytes (Figures 2A and 2B). Whilst sections from group III showed positive iNOS immunoreactivity within the cytoplasm of nearly all hepatocytes (Figure 2C). Sections from group IV appeared almost as groups I and II (Figure 2D).

Toluidine blue stained semithin sections: Semithin sections of livers from both groups I and II showed hepatocytes with vesicular nuclei enclosing blood sinusoids. Some hepatocytes demonstrated cytoplasmic vacuoles. Von Kupffer cells exhibiting eccentric dark nuclei were demonstrated lining the blood sinusoids (Figures 3A and 3B). Group III showed hepatocytes with small dark nuclei, karyorrhexed

\begin{tabular}{|c|c|c|c|c|}
\hline & Group I & Group II & Group III & Group IV \\
\hline AST & $86.4 \pm 2.1486$ & $86.3 \pm 2.1486$ & $187.2 \pm 2.1486^{*}$ & $90.1 \pm 2.1486$ \\
\hline ALT & $41.7 \pm 1.2736$ & $42.1 \pm 1.2736$ & $91.1 \pm 1.2736^{*}$ & $43.7 \pm 1.2736$ \\
\hline MDA & $1.106 \pm 0.259$ & $1.042 \pm 0.259$ & $11.555 \pm 0.259^{*}$ & $1.154 \pm 0.259$ \\
\hline GSH & $51.228 \pm 0.8777$ & $52.945 \pm 0.8777$ & $20.24 \pm 0.8777^{*}$ & $50.224 \pm 0.8777$ \\
\hline
\end{tabular}

Table 1: The mean values of serum AST, ALT (U/ I), MDA (nmol /ml) and GSH $(\mathrm{mmol} / \mathrm{ml}) \pm \mathrm{SEM}$ in the studied groups. 
Citation: Abd El-Fattah LI, Ibrahim Ismail D (2015) Histological Study on the Protective Effect of Green Tea Extract on the Liver of Rats Exposed to Ketamine. J Cytol Histol 6: 349. doi:10.4172/2157-7099.1000349
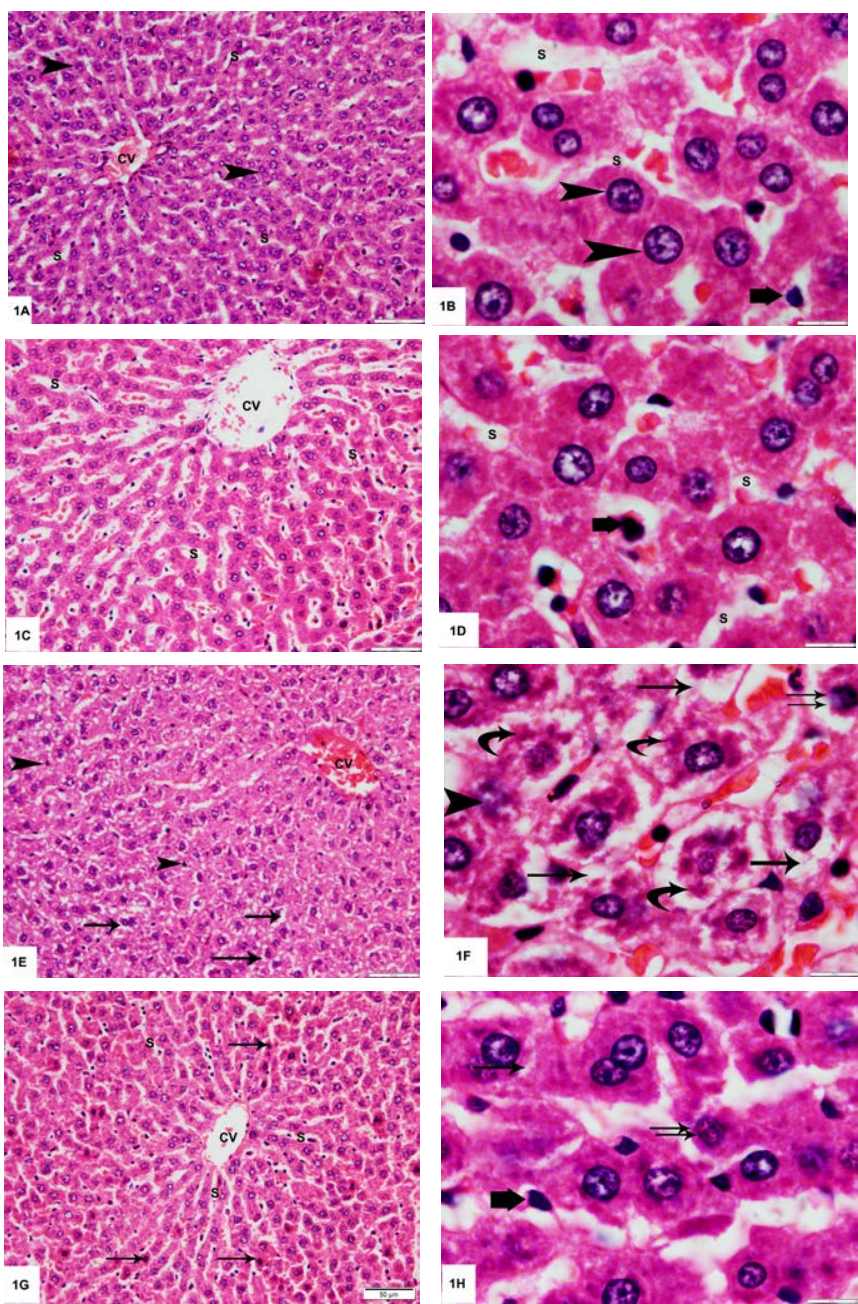

Figure 1: Photomicrographs of sections in rats' livers stained with $\mathrm{H}$ and $\mathrm{E}$ A. Group I (control group) shows a central vein (CV) with radiating cords of hepatocytes; most of them are mononucleated some are binucleated (arrowheads).Cords of hepatocytes enclose blood sinusoids (S) in between 200X. B. A higher magnification showing hepatocytes with acidophilic cytoplasm and central rounded vesicular nuclei (arrowheads). Blood sinusoids (S) are seen in between the cords of hepatocytes. Von Kupffer cells with dark nuclei are demonstrated (Thick arrow) 1000X. C. Group II (green tea group) demonstrates central vein (CV) with radiating hepatocytes. Note the blood sinusoids (S) 200X. D. A higher magnification showing the hepatocytes with blood sinusoids (S) in between. Note Von Kupffer cell (Thick arrow) 1000X. E. Group III (Ketamine group) reveals congested central vein (CV). Hepatocytes show cytoplasmic vacuolization (arrows). Some hepatocytes demonstrate dark nuclei (arrowheads) 200X. F. A higher magnification of group III showing hepatocytes with cytoplasmic vacuolization (arrows). Note the karryorhexed (arrowhead) and the small nuclei (double arrows). Deeply acidophilic fragmented cytoplasmic bodies are also noted (curved arrows) 1000X. G. Group IV (Ketamine and green tea group) shows central vein (CV) with radiating hepatocytes enclosing blood sinusoids (S) in between. Note some hepatocytes with deep acidophilic cytoplasm and dark small nuclei (arrows) 200X. H. A closer observation to the hepatocytes showing minimal cytoplasmic areas of vacuolization (arrow) and other with small nucleus (double arrow). No acidophilic fragmented bodies could be detected. Von Kupffer cell could be noted (Thick arrow) 1000X.

nuclei, no nuclei, or multiple cytoplasmic vacuoles. Apparently dilated blood sinusoids with widely separated endothelial cell from the adjacent hepatocytes were noted (Figure 3C). Regarding sections from group IV, they demonstrated histological appearance almost similar to the control group, except few hepatocytes exhibiting small dark nuclei and few small cytoplasmic vacuoles. Some blood sinusoids appeared comparable to those of the control group (Figure 3D).

Electron microscopic examination: Ultrathin sections from groups I and II showed adjacent hepatocytes exhibiting euchromatic nuclei with regular outline, many mitochondria, rough endoplasmic reticulum, and smooth endoplasmic reticulum. Except for few
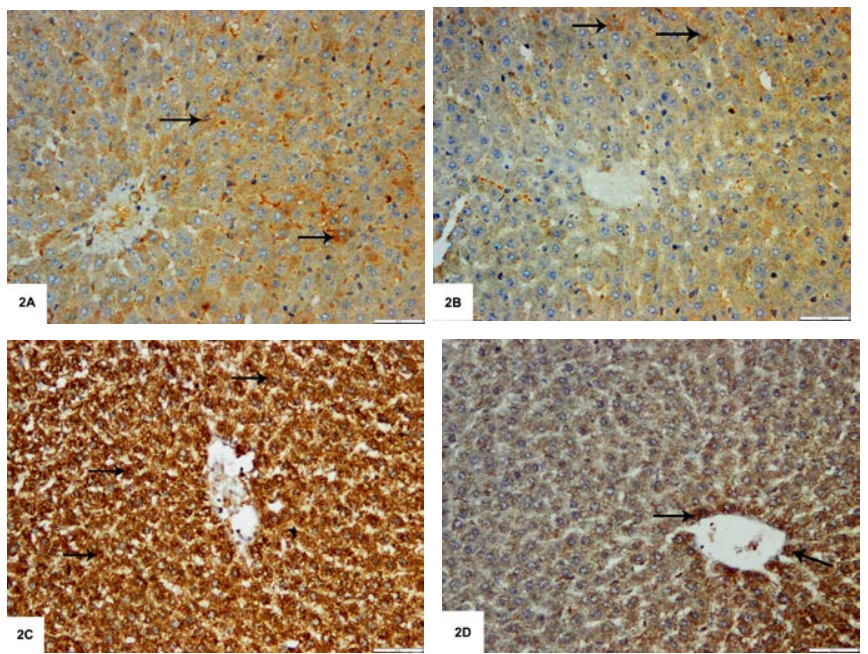

Figure 2: Photomicrographs of sections of rats' livers stained with anti- iNOS antibody, 200X. A. The control group shows positive iNOS immunoreactivity within the cytoplasm of some hepatocytes (arrows). B. Group II demonstrates positive iNOS immunoreaction within the cytoplasm of some hepatocytes (arrows). C. Group III reveals positive iNOS immunoreactivity within the cytoplasm of nearly all hepatocytes (arrow). D. Group IV shows positive iNOS immunoreactivity within the cytoplasm of some hepatocytes (arrows).
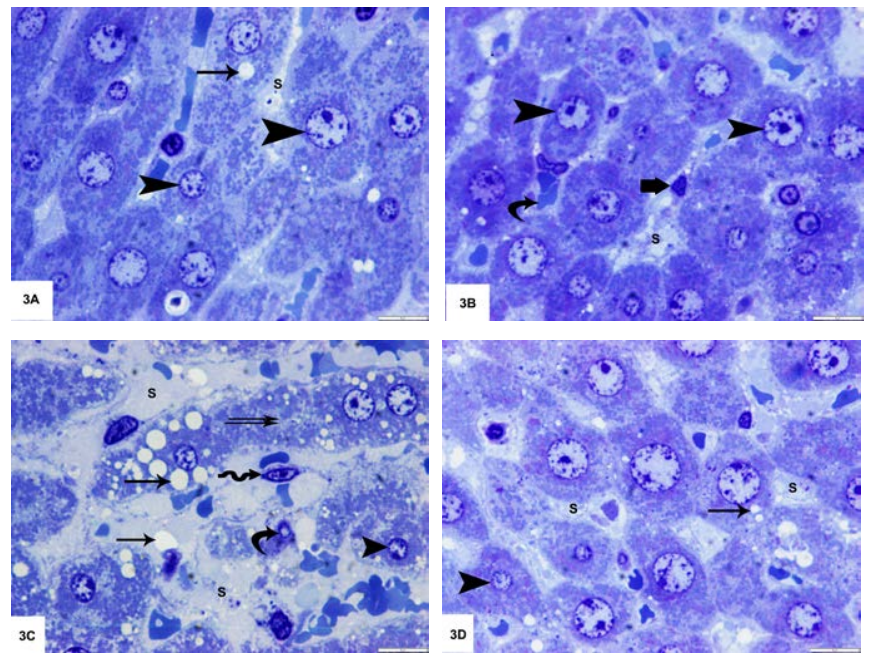

Figure 3: Photomicrographs of semithin sections of rats' livers stained with toluidine blue, 1000X. A. The control group shows hepatocytes with vesicular nuclei (arrowheads) and blood sinusoids (S). Note a cytoplasmic vacuole within a hepatocyte (arrow). B. Group II reveals hepatocytes with vesicular nuclei (arrowheads) and the blood sinusoids (S). VonKupffer cell (thick arrow) and RBCs (curved arrow) are also seen. C. Group III shows hepatocytes with apparently small nuclei (arrowhead), multiple cytoplasmic vacuoles of variable sizes (arrows). A hepatocyte with karyorrhexed nucleus (curved arrow) and another with absent nucleus could be observed (double arrow). Note dilated blood sinusoids (S) and the endothelial cell (zigzag arrow) widely separated from the adjacent hepatocyte. D. Group IV demonstrates few hepatocytes with small nuclei (arrowhead); otherwise nuclei are comparable to control. The cytoplasm has few small vacuoles (arrow). Note blood sinusoids (S) apparently comparable to control. 
Citation: Abd El-Fattah LI, Ibrahim Ismail D (2015) Histological Study on the Protective Effect of Green Tea Extract on the Liver of Rats Exposed to Ketamine. J Cytol Histol 6: 349. doi:10.4172/2157-7099.1000349

electron-lucent vacuoles were detected in group II (Figures $4 \mathrm{~A}$ and 4B). Hepatocytes from group III exhibited small shrunken nuclei with irregular outline. Nuclei showed condensed peripheral heterochromatin and focal areas of widened inter membranous space of nuclear envelope. There were multiple areas of cytoplasmic vacuolization around the nuclei and in relation to mitochondria. Cisternae of rough endoplasmic reticulum exhibited discontinuity in some fields and appeared dilated in other fields. Mitochondria were separated by empty spaces or dilated and discontinued rER (Figure 4C). Group IV showed hepatocytes exhibiting euchromatic nuclei with regular outline, many mitochondria separated by regular rER. Few dilated rER and electron lucent vacuoles of variable sizes were noticed (Figure 4D).

Morphometric results: Mean area percent of iNOS positive immunoreactivity showed statistically significant increase $(\mathrm{P}<0.05)$ in group III (Ketamine) in comparison to other groups. However, groups II and IV were comparable to control (Table 2).

\section{Discussion}

There is paucity of studies focusing on the potential toxic effects of ketamine on hepatocytes. Nevertheless, Navarro and Senio [9] reported
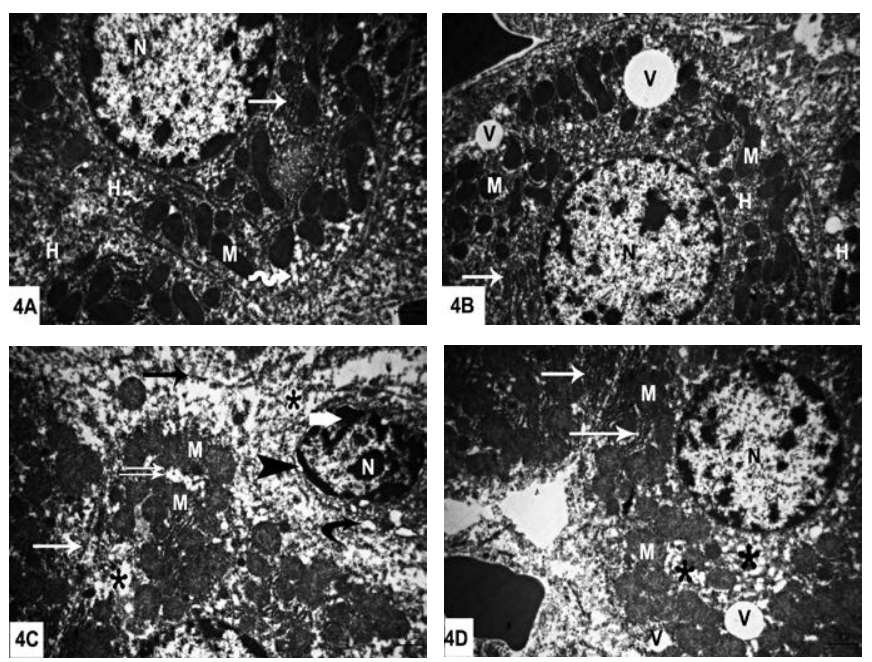

Figure 4: Electron photomicrographs of rats' livers, TEM, X8000. A. The control group shows two adjacent hepatocytes $(\mathrm{H})$. One cell exhibits euchromatic nucleus with regular outline $(\mathrm{N})$, many mitochondria $(\mathrm{M})$, rough endoplasmic reticulum (arrow) and smooth endoplasmic reticulum (zigzag arrow). B. Group II demonstrates two neighboring hepatocytes $(\mathrm{H})$. The hepatocyte has euchromatic nucleus $(\mathrm{N})$, many mitochondria $(\mathrm{M})$ and rough endoplasmic reticulum (arrow). Note two electron lucent vacuoles of variable sizes (V). C. Group III shows hepatocyte with shrunken nucleus with irregular outline $(\mathrm{N})$, condensed peripheral heterochromatin (thick arrow) and focal areas of widened inter membranous space of nuclear envelope (arrowhead). Note multiple areas of cytoplasmic vacuolization (stars) around the nucleus and in relation to mitochondria. Cisternae of rough endoplasmic reticulum appear exhibiting discontinuity (arrow) or dilated (curved arrow). Mitochondria (M) are separated by empty spaces (double arrow) or discontinued rER (white arrow). D. Group IV shows hepatocyte exhibiting euchromatic nucleus with regular outline $(\mathrm{N})$, many mitochondria (M) separated by regular (arrow) or widened rER (stars). Note two electron lucent vacuoles (V) of variable sizes with the smaller one near the cell surface.

\begin{tabular}{|r|r|r|r|r|}
\hline & Group I & Group II & Group III & Group IV \\
\hline \multirow{2}{*}{$\%$ iNOS } & $4.592 \pm 0.978$ & $4.067 \pm 0.978$ & $\begin{array}{c}16.195 \pm \\
0.978^{*}\end{array}$ & $7.073 \pm 0.978$ \\
\hline
\end{tabular}

*significantly different from the value of the other groups at $P<0.05$

Table 2: The mean area percent of iNOS positive immunoreactivity \pm SEM in the studied groups. the hepatotoxicity of ketamine during pain management. Green tea, from the plant Camellia sinensis, proved to have health benefits in both humans and animals [10].

Accordingly, the current study was planned to explore the potential hepatotoxicity of ketamine, as well as, the possible hepatoprotective effect of green tea. This was carried out at histological and biochemical levels.

Biochemically, the liver function was assessed by demonstrating the serum levels of AST and ALT. Furthermore, the serum levels of Glutathione (GSH) and malondialdehyde (MDA), as indicators of oxidative stress, were also detected. The present work demonstrated statistically significant increase in the levels of AST, ALT, and MDA, and a significant decrease in the level of GSH in ketamine treated rats in comparison to other groups; including green tea and ketamine green tea treated rats which were comparable to control. This might suggest the hepatotoxic effect of ketamine, as well as the possible hepatoprotective effect of green tea. Moreover, it could highlight the mechanism underlying the green tea hepatoprotective effect; being an antioxidant.

Such findings are concomitant with the findings of Kalkan et al. [1] who reported that ketamine alters liver function tests. Moreover, Chacko et al. [10] stated that green tea intake reduced the marker of oxidative stress MDA.

$\mathrm{H} \& \mathrm{E}$ stained sections of the current work demonstrated normal histological picture in both the control and green tea groups. The ketamine treated rats revealed many histological changes at light microscopic level including congested central veins. This finding might be a reaction to increase the blood supply as an attempt to overcome the injurious effect of ketamine or a response to the released cytokines following hepatocytes injury.

Vacuolated hepatocytes exhibiting dark small nuclei, indicative of apoptosis, with acidophilic fragmented bodies were demonstrated. Apoptosis of hepatocytes could be due to massive oxidative stress generated by ketamine. This finding is supported by Kalkan et al. [1] reporting that ketamine induces apoptotic cell deaths associated with mitochondrial degeneration in hepatic cells. The acidophilic fragmented bodies detected in the present work could be Mallory bodies. They have been associated with a variety of liver diseases [11].

Regarding the green tea and ketamine treated group, liver sections were comparable to the control. Such improvement of the histological picture might be due to the antioxidant effect of green tea. This hypothesis is so far supported by the reports that green tea prevents hepatotoxicity [12]. The health-promoting effects of green tea are mainly attributed to its polyphenol content (catechin). Catechins reduced the formation of peroxides more effectively than alpha-tocopherol [10].

Concerning the immunohistochemically stained liver sections, the current work recorded positive iNOS immunoreactivity in all the studied groups with statistically significant increased immunoexpression in the ketamine treated group compared to other groups. This is consistent with previous studies of Vakkala et al. [13] reporting iNOS in many cells including hepatocytes. Moreover, the increase recorded in ketamine group could be due to the generation of reactive species derived from oxygen and nitrogen, which could interfere with the antioxidant defense system. Hand in hand, it was found that induction of iNOS is associated with high concentrations of reactive nitrogen metabolites [13].

No significant difference was detected between control and green 
tea -ketamine treated group. This might be attributed to the antioxidant and anti-inflammatory properties of green tea as previously mentioned. Green tea was reported as an anti-inflammatory agent inhibiting cyclooxygenase 2 (COX-2) enzymatic activity decreasing liver injury, as well; green tea polyphenols reduce (iNOS) gene expression and inhibit enzyme activity [14].

Concerning semithin sections stained with toluidine blue, liver sections of groups I and II demonstrated few cytoplasmic vacuoles most probably of lipid content. This might be related to dietary causes. The liver sections of ketamine treated group revealed many pathological changes including hepatocytes with karryorrhexed nuclei. This is might represent cellular necrosis that could be attributed to hepatotoxic effect of ketamine. In consistent, hepatocyte damage has been reported to be associated with ketamine treatment [15]. Hand in hand, the El-Sayyad et al. [16] reported that in hepatotoxicity induced by chemicals, necrosis occurs following or currently with apoptosis. Moreover, liver sections of the current work demonstrated multiple cytoplasmic vacuoles of variable sizes. Those vacuoles could be of lipid content associated with increased lipid peroxidation as a sequel of the toxic effect of ketamine. Tarantola et al. [17] reported unstained lipid droplets presumed to be of saturated lipids unstained by osmium tetroxide $\left(\mathrm{OsO}_{4}\right)$ and associate their abundance with scarce hepatocyte activity. Furthermore, ketamine induces metabolic effects including changes in lipid metabolism in liver that may be related to hepatotoxicity [15].

Apparently dilated blood sinusoids were noted with widely separated endothelial cells from the adjacent hepatocytes. This could be explained on the basis of lipid peroxidation that might affect the cell membrane, causing membrane disruption, of different cells including the endothelial cells. Moreover, the dilated blood sinusoid might be a sequel of increased iNOS reported in our study. The increased iNOS causes increased production of $\mathrm{NO}$ causing vasodilatation of the sinusoids. 13. Vakkala et al. [13] reported NO as a vasodilator. Other studies reported ketamine to affect vascular endothelial cells of the liver with significant sinusoidal dilatation [1].

The liver sections of ketamine treated with green tea revealed a picture almost comparable to control. This could be explained on the basis of anti-oxidative and anti-inflammatory effects of green tea which, by its polyphenols content, could prevent excessive lipid peroxidation. This hypothesis could be supported by Chacko et al. [10] who stated that green tea significantly decrease lipid peroxidation. Moreover, Shimizu et al. [18] reported that the anti-oxidative and anti-inflammatory activities are the key mechanisms of green tea catechins (polyphenols).

The recorded blood sinusoids, apparently comparable to those of the control, in ketamine and green tea treated group of the current work could be attributed to the iNOS reducing capacity of the green tea, as previously mentioned, resulting in decrease in NO with its vasodilating effect.

Regarding the ultrastructural results of the current study, the green tea group and the ketamine green tea treated groups were almost comparable to control. Whereas, the ketamine treated group demonstrated many changes of hepatocytes including small irregular nuclei, with condensed peripheral chromatin and focal areas of widened inter membranous space of nuclear envelope. The small nuclei with disturbed chromatin distribution could be a part of the apoptotic nuclear change caused by ketamine. Moreover, the changes within the nuclear envelope might be due to changes in phospholipid content of the membrane as a part of the oxidative hepatocyte injury associated with changes in lipid metabolism induced by ketamine as previously suggested.
Furthermore, in the present work, the ultrastructure of the hepatocyte cytoplasm of the ketamine treated group revealed dilatation and discontinuity of rER with multiple areas of cytoplasmic vacuolization of damaged hepatocytes. Such finding could be attributed to the massive oxidative stress affecting hepatocytes and caused by ketamine. This might be associated with radicals attacking lipids of endoplasmic membrane causing its dilatation and disruption, as supported by Helal et al. [7]. Csala et al. [19] reported that dilatation of the endoplasmic reticulum is representative of severely damaged hepatocyte. Helal et al. [7] added that, free radicals lead to lipid peroxidation which often associates massive hepatocyte necrosis. This hypothesis goes hand in hand with our finding in the ketamine - green tea treated group as green tea is a well known antioxidant that decreases oxidative stress and lipid peroxidation as was previously mentioned.

In conclusion, the present study identified pathological features at both biochemical histological levels in the rat liver treated with ketamine. Since the efficacy of a hepatoprotective drug could be judged through its capacity to restore or maintain the normal hepatic function that have been distorted by the hepatotoxic agent, the current study provide an evidence of the possible hepatoprotective effect of green tea against the hepatotoxic effect of ketamine.

Human investigations are recommended to be carried out to demonstrate whether similar results could be obtained.

\section{References}

1. Kalkan Y, Tomak Y, Altuner D, Tumkaya L, Bostan H, et al. (2014) Hepatic effects of ketamine administration for 2 weeks in rats. Hum Exp Toxicol 33: $32-40$.

2. Chen JH, Tipoe GL, Liong EC, So HS, Leung KM, et al. (2004) Green tea polyphenols prevent toxin-induced hepatotoxicity in mice by down-regulating inducible nitric oxide-derived prooxidants. Am J Clin Nutr 80: 742-751.

3. Lambert JD, Kennett MJ, Sang S, Reuhl KR, Ju J, et al. (2010) Hepatotoxicity of high oral dose (-)-epigallocatechin-3-gallate in mice. Food Chem Toxicol 48: 409-416.

4. Ramadan G, El-Beih NM, Abd El-Ghffar EA (2009) Modulatory effects of black vs. green tea aqueous extract on hyperglycaemia, hyperlipidaemia and liver dysfunction in diabetic and obese rat models. Br J Nutr 102: 1611-1619.

5. Kiernan JA (2001) Histological and histochemical methods: theory and practice. (3rdedn.) Arnold Publisher, London, New York and New Delhi.

6. Mazroa SA, Askar SA, El-Shafey S (2009) Immunohistochemical distribution of inducible nitric oxide synthas enzyme (iNOS) in the renal cortex of contro and diabetic adult male albino rats and the effect of iNOS inhibition by aminoguanidine. Egypt J Histol 32: 235-245.

7. Helal OK, Mansy AE, Hassan NA (2001) Does Commiphora extract have an effect on carbon tetrachloride-induced liver injury in adult albino rats? Histological and biochemical study. The Egyptian Journal of Histology 36: 528-536.

8. Wilcox PR (2009) Basic statistics: Understanding conventional methods and modern insights (1stedn.) Oxford University Press, Oxford, New York.

9. Navarro VJ, Senior JR (2006) Drug-related hepatotoxicity. N Engl J Med 354: 731-739.

10. Chacko SM, Thambi PT, Kuttan R, Nishigaki I (2010) Beneficial effects of green tea: A literature review. Chin Med 5: 13.

11. Zatloukal K, French SW, Stumptner C, Strnad P, Harada M, et al. (2007) From Mallory to Mallory-Denk bodies: what, how and why? Exp Cell Res 313: 20332049.

12. Crespy V, Williamson G (2004) A review of the health effects of green tea catechins in in vivo animal models. J Nutr 134: 3431 S-3440S.

13. Vakkala M, Kahlos K, Lakari E, Pa"a"kko“ P, Kinnula V, et al. (2000) Inducible Nitric Oxide Synthase Expression, Apoptosis, and Angiogenesis in in Situ and Invasive Breast Carcinomas. Clinical Cancer Research 6: 2408-2416.

14. Ibrahim AAE, Saleh HE, El-Shinnawy NA (2008) The role of ginger or green 
Citation: Abd El-Fattah LI, Ibrahim Ismail D (2015) Histological Study on the Protective Effect of Green Tea Extract on the Liver of Rats Exposed to Ketamine. J Cytol Histol 6: 349. doi:10.4172/2157-7099.1000349

tea in counteracting the deleterious effect of benzene sulfonic acid in weanling male rats. Egyptian Journal of Natural Toxins 5: 56-99.

15. Saranteas T, Zotos N, Lolis E, Stranomiti J, Mourouzis C, et al. (2005) Mechanisms of ketamine action on lipid metabolism in rats. Eur $\mathrm{J}$ Anaesthesio 22: $222-226$

16. El-Sayyad HI, Ismail MF, Shalaby FM, Abou-El-Magd RF, Gaur RL, et al. (2009) Histopathological effects of cisplatin, doxorubicin and 5-flurouracil (5-FU) on the liver of male albino rats. Int J Biol Sci 5: 466-473.
17. Tarantola E, Bertone V, Milanesi G, Capelli E, Ferrigno A, et al. (2012) Dipeptidylpeptidase--IV, a key enzyme for the degradation of incretins and neuropeptides: Activity and expression in the liver of lean and obese rats. Eur J Histochem 56: e41.

18. Shimizu M, Shirakami Y, Sakai H, Kubota M, Kochi T, et al. (2015) Chemopreventive potential of green tea catechins in hepatocellular carcinoma. Int J Mol Sci 16: 6124-6139.

19. Csala M, Bánhegyi G, Benedetti A (2006) Endoplasmic reticulum: a metabolic compartment. FEBS Lett 580: 2160-2165. 